Research

\title{
Cyberbullying, metacognition, and quality of life: preliminary findings from the Longitudinal Adolescent Brain Study (LABS)
}

\author{
Larisa T. McLoughlin ${ }^{1}$. Gabrielle Simcock ${ }^{1}$. Paul Schwenn ${ }^{1}$. Denise Beaudequin ${ }^{1}$. Christina Driver ${ }^{1}$. \\ Lee Kannis-Dymand ${ }^{1}$. Jim Lagopoulos ${ }^{1}$. Daniel F. Hermens ${ }^{1}$
}

Received: 2 November 2021 / Accepted: 20 December 2021

Published online: 12 January 2022

(c) The Author(s) 2022 OPEN

\begin{abstract}
Cyberbullying contributes to poor mental health outcomes and quality of life (QoL), and peer victimisation has been shown to be positively associated with both positive and negative metacognition. Whilst metacognitive beliefs are associated with pathological worry, obsessive-compulsive symptoms, and rumination in depression, research is yet to examine whether metacognitive beliefs influence negative outcomes, such as reduced QoL, associated with experiences of cyberbullying. This study examines whether cybervictimisation, cyberbullying and metacognition play predictive roles in QoL, and if metacognition mediates any association between cybervictimisation, cyberbullying and QoL over time. Participants in the Longitudinal Adolescent Brain Study (LABS), aged 12 years and in grade 7, who had completed up to four assessment time-points (T) were included in this analysis: T1: $N=65 ; T 2: N=61 ; T 3: N=56 ; T 4: N=44$. Structural equation modeling revealed significant associations between cyberbullying, cybervictimisation, metacognitive beliefs, and QoL. However, mediation analysis showed that only cognitive confidence acted as a partial mediator between cybervictimisation and QoL. The results suggest those who were more frequently cybervictimised had reduced confidence in their memory, which resulted in lower QoL. In addition, uncontrollability/danger and superstition, punishment, and responsibility were all negatively associated with QoL, indicating that as these metacognitive beliefs increased, QoL decreased. Our findings highlight the detrimental impact that cybervictimisation experiences can have on QoL in young people over time, and that some forms of metacognitive beliefs can also impact QoL. These findings can inform educators and health professionals on the importance of metacognition in regard to QoL over time, particularly in those who experience cybervictimisation.
\end{abstract}

Keywords Cyberbullying · Cybervictimisation · Longitudinal · Metacognition · Quality of life · Mediation · Longitudinal Adolescent Brain Study

\section{Introduction}

Cyberbullying is a significant concern for adolescents and is a contributor to mental illness $[3,11,19,23,25,26,33$, 37 in this age group. Cyberbullying has been defined as perpetration of aggressive, repeated, intentional acts that aim to threaten, harass, or embarrass another individuals, carried out via electronic means [32]. The term cybervictimisation will be used throughout this paper to describe the experience of those being cyberbullied. In addition,

Larisa T. McLoughlin, Imclough@usc.edu.au | ${ }^{1}$ Thompson Institute, University of the Sunshine Coast, 12 Innovation Parkway, Birtinya, QLD, Australia.

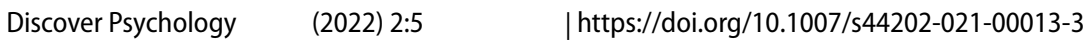


research shows that young people who have experiences of cyberbullying report significantly lower on quality of life (QoL) measures [13, 14]. The World Health Organisation (WHO) ([15], p. 1405) defines QoL as "an individuals' perception of their position in life in the context of the culture and value systems in which they live and in relation to their goals, expectations, standards and concerns. It is a broad ranging concept affected in a complex way by the persons' physical health, psychological state, level of independence, social relationships and their relationship to salient features of their environment".

Whilst metacognitive beliefs have been found to be associated with pathological worry, obsessive-compulsive symptoms, and rumination in depression $[5,28,35,46]$, research is yet to examine whether metacognitive beliefs influence the negative outcomes associated with experiences of cyberbullying, such as QoL. Metacognitive knowledge and abilities develop during early adolescence and allow individuals the capacity to understand and reflect on their emotions [22]. Research regarding metacognitive beliefs and its association with cyberbullying and cybervictimisation is lacking. Utilising preliminary exploratory findings from the Longitudinal Adolescent Brain Study (LABS), the present paper sought to explicate the associations between cyberbullying and cybervictimisation, metacognition, and QoL.

Victims of both traditional bullying and cyberbullying experience negative mental health outcomes, including; low self-esteem, depression, anxiety and stress, social and behavioural difficulties, lower QoL and suicidal thoughts or behaviours $[13,14,16,21,29]$. Further research has indicated that cyberbullying perpetration results in increased psychological distress, as well as increased maladaptive behaviours, such as substance abuse [36], and decreased QoL [13, 14].

Given the multi-dimensional nature of QoL, WHO identify six broad domains: (a) physical; (b) psychological; (c) level of independence; (d) social relationships; (e) environment; and (f) spirituality/religion/personal beliefs ([15], p. 1405). Researchers highlight that when evaluating QoL, metacognition is an important variable to consider because impaired metacognition can impact ability to assess QoL [2], yet to date, very little research has been conducted to address this relationship specifically, and no research has examined associations in an adolescent sample in relation to cyberbullying.

Research suggests that adolescents as young as 12 have the capacity for metacognitive beliefs [4, 10]. Metacognition or our 'beliefs about thinking' (i.e., metacognitive beliefs) refer to the importance of thoughts, and the events and processes that are involved in the control, modification, and interpretation of thinking [24, 41, 42]. The metacognitive model of psychological disorder posits that anxiety and depression result from perseverative thinking, such as worry and rumination, that is driven by metacognitive beliefs $[41,45]$. It has been established that individuals with major depressive disorder and generalized anxiety disorder respond to negative thoughts with sustained rumination or worry respectively, because they hold positive beliefs about the value of such processes (i.e., that worry or rumination is beneficial); likewise, they hold negative beliefs that this process is beyond their control (i.e., worry or rumination is uncontrollable or harmful) $[42,45,46]$. This theory is relevant in regard to cyberbullying, as young people who have been cyberbullied experience worry and rumination [9, 38].

Positive and negative metacognitive beliefs are a transdiagnostic factor across a wide range of psychological disorders $[43,46]$. Positive metacognitive beliefs refer to the perceived helpfulness or benefits of engaging in repetitive negative thinking such as "I must worry in order to be prepared", and "Thinking the worst will keep me safe". Negative metacognitive beliefs focus on the uncontrollability, dangerousness, and consequences of repetitive negative thinking such as "I cannot control my thoughts" and "If I have violent thoughts, I will act on them against my will" [44]. To date, scant research has examined the links between metacognition and cyberbullying, however, a recent study of 1169 Italian adolescents found that peer victimisation was positively associated with both positive and negative metacognition [12]. In addition, in this study metacognition was linked to worry and rumination and was associated with higher psychological and somatic problems [12].

To our knowledge, there are no prospective longitudinal studies investigating temporal relationships between cyberbullying, metacognition and QoL in young adolescents. Further research is therefore needed to examine the unique associations between these inter-related variables. The current study aimed to address these gaps in the literature by examining whether cyberbullying and metacognition play predictive roles in QoL, and if metacognition mediated any association between cybervictimisation, cyberbullying and QoL. We hypothesised that:

1. Cyberbullying and cybervictimisation would negatively predict QoL, and positively predict all metacognition subscales

2. Metacognitive beliefs would negatively predict QoL 
3. Metacognitive beliefs would mediate the association between cyberbullying and cybervictimisation and QoL over time.

\section{Method}

\subsection{Study design and procedure}

LABS is a prospective cohort study launched in the Sunshine Coast region of Australia in August 2018. The study recruits young people aged 12 years in grade 7, with follow-up assessments every four months over a 5-year period, totalling 15 assessment time-points for each participant. At each time-point, assessments include a self-report questionnaire on social-emotional wellbeing, a battery of cognitive assessments, a neuropsychiatric interview, and multimodal neuroimaging scans (EEG, MRI). In the current study we report data taken from the self-report questionnaire completed as part of LABS assessments relevant to the study hypotheses at time-point 1 (T1- first appointment) through to time-point 4 (T4).

Assessments were completed at the Thompson Institute (TI), USC. Participants completed the self-report questionnaire on a touch-screen tablet, using the Qualtrics survey platform (Copyright $\odot 2019$ Qualtrics, Provo, UT, USA), in the presence of the researcher. The self-report questionnaire took approximately $30 \mathrm{~min}$ to complete. Participants received a \$20 gift voucher as reimbursement at each time-point. Please note, all self-report measures were tested for reliability and validity (Cronbach's alpha) and were found to be satisfactory and consistent with previous research.

\subsection{Participants}

\subsubsection{Recruitment}

Participants were recruited into LABS via advertising at local community events and via social media. Ongoing recruitment has continued since the commencement of the study in 2018.

\subsubsection{Inclusion and exclusion criteria}

Young people aged 12 years and in grade 7 were included in the study. Young people suffering from a major neurological disorder, intellectual disability, major medical illness, or who had sustained head injury (with loss of consciousness for more than $30 \mathrm{~min}$ ) were excluded from the study. This paper uses data from participants who had completed the first four time-points of the study. Table 1 displays demographic information, and numbers of participants in each time-point. This equates to over 200 time-points of data.

\subsubsection{Cyberbullying self-report measure}

The Berlin Cyberbullying-Cybervictimisation Questionnaire $(B C y Q, 19$ items for cybervictimisation and 20 items for cyberbullying) was used to assess experiences of cyberbullying and cybervictimisation [30, 31]. Participants were asked if they had experienced a list of behaviors over the previous 6-month period, as well as if they had acted in that way. The scale ranged from 1 "has not happened to me at all", to 5 "several times a week". Aggregated scores were then created to allow a total score for both cyberbullying (score range $=18-90$ ) and cybervictimisation (score range $=17-85$ ), with higher scores indicating greater frequency of either bullying or victimisation.

Table 1 Demographic details

\begin{tabular}{lllll}
\hline & T1 $(\mathrm{N}=65)$ & $\mathrm{T} 2(\mathrm{~N}=61)$ & $\mathrm{T} 3(\mathrm{~N}=56)$ & $\mathrm{T4}(\mathrm{N}=44)$ \\
\hline Male $(n)$ & 35 & 32 & 30 & 24 \\
Female $(n)$ & 30 & 29 & 26 & 20 \\
Age $(\mathrm{M} \pm \mathrm{SD})$ & $12.6 \pm 0.3$ & $12.9 \pm 0.3$ & $13.3 \pm 0.3$ & $13.7 \pm 0.3$ \\
\hline
\end{tabular}




\subsubsection{Metacognition self-report measure}

The Metacognition Questionnaire-Adolescent version (MCQ-A, 30 items) was used to assess individual differences in metacognitive beliefs, judgements, and monitoring tendencies [4, 44]. The MCQ-A consists of five subscales: (1) positive metacognition (i.e., the belief that worrying may be helpful in preventing or avoiding problems in the future); (2) negative metacognition (i.e., beliefs that worry is harmful, dangerous, and uncontrollable); (3) cognitive confidence (i.e., assessing low confidence in memory and attention); (4) superstition, punishment, and responsibility (SPR) (i.e., need for control, beliefs concerning the need to control one's thoughts and being responsible for the negative consequences of not doing so); (5) cognitive self-conscious (i.e. extent to which a person is aware of, and focuses on, their thinking; assesses the tendency to monitor one's own thoughts and focus attention inwards). All items are rated on a four-point Likert scale, from 1 (not at all) to 4 (totally). Total scores for each subscale are calculated from the 6 items of each subscale, yielding total scores of 6-24. A total score is achieved by summing the subscales, ranging from 30 to 120 . Higher total scores are indicative of higher levels of metacognitive beliefs or processes. In other words, higher scores indicate more positive and negative beliefs about worry, reduced confidence in memory, greater belief in the need to control thoughts and an increased tendency towards self-focused attention.

\subsubsection{Quality of life self-report measure}

The World Health Organization Quality of Life (WHOQOL-BREF) (26 items) measures the following broad domains: physical health, psychological health, social relationships, and environment. Total scores are achieved for each domain, measured with various anchors on a scale of 1 to 5 , with higher scores indicating better quality of life in the domain. The WhOQOL-BREF has been employed extensively in both psychological and medical research as a crosscultural measure of health-related quality of life and with adolescents [6].

\subsection{Data analysis}

Data were analysed using SPSS version 26 [8] and AMOS [1]. Analysis included using SPSS for descriptive statistics to examine age and gender (Table 1), scale reliability (Table 2), and AMOS for the Structural Equation Modelling (Figs. 1, $2,3,4)$. Data was examined for outliers, linearity, and multicollinearity. Bootstrapping was applied to the SEM to account for the non-normal distribution (see below).

\subsection{Structural equation modelling (SEM)}

SEM was conducted to determine the predictive nature of the associations between cyberbullying and cybervictimisation and QoL, and if the different forms of metacognition would mediate this. SEM was conducted using data from T1

Table 2 Standard deviation (SD), reliability coefficient (Cronbach's alpha), unstandardised factor loadings and error variances for composite variables in SEM for T4 aggregates

\begin{tabular}{lllll}
\hline Name & SD & $\begin{array}{l}\text { Reliability coef- } \\
\text { ficient } \\
\mathrm{a}\end{array}$ & Factor loading & Error variance \\
\hline Positive Beliefs & 3.49 & 0.93 & 3.36 & 0.91 \\
Uncontrollability/danger & 3.56 & 0.85 & 3.28 & 1.90 \\
Cognitive confidence & 3.37 & 0.84 & 3.08 & 1.85 \\
SPR & 2.88 & 0.68 & 2.38 & 2.66 \\
Cognitive self-consciousness & 4.60 & 0.86 & 4.26 & 2.96 \\
Physical QoL & 3.58 & 0.89 & 3.39 & 1.36 \\
Psychological QoL & 4.29 & 0.87 & 3.99 & 2.45 \\
Social QoL & 1.46 & 0.88 & 1.37 & 0.26 \\
Environmental QoL & 4.14 & 0.87 & 3.87 & 2.16 \\
\hline
\end{tabular}

Cronbach's alpha was used to determine scale reliability 
(cyberbullying and cybervictimisation) through to T4 (QoL and metacognition), thereby exploring associations between variables across the first year of LABS. The four domains of the WHOQOL were inter-correlated, and so these were included in the models as an overall QoL output variable. The five dimensions of metacognition were included independently, as they were not correlated.

Mediation analyses were conducted using SEM, with cyberbullying and cybervictimisation as the causal (predictor/ independent) variables, QoL as the outcome/dependent variable and the different domains of metacognition as the mediating variable. In order to consider and include the aggregated constructs of QoL and metacognition at T4, a parameter estimates calculator [17] was used to determine factor loadings and error variance (Table 2). All factor loadings were considered satisfactory (greater than 0.9) [34].

To account for non-normal distribution of the data, bootstrapping was performed for each of the models with 2000 samples and bias-corrected confidence intervals set to $95 \%$. Chin's [7] guidelines were used, which suggests that standardised regression weights should be $0.20-0.30$ or higher to be meaningful.

\section{Results}

\subsection{SEM}

Correlations revealed that the QoL domains were all inter-correlated, therefore covariances were drawn between each of the variables in the models to include $Q$ Q L as a single outcome variable. When running the first model, it was noted that one inter-item correlation was not significant (between physical and psychological), therefore this correlation was removed. Note that in each model figure, rectangles in models denote measured variables, and ovals denote latent variables; single-headed arrows represent regression paths and double-headed arrows represent correlations; ' $\mathrm{e}$ ' variables represent error variances, and ' $z$ ' variables represent residuals for latent variables. Only significant regression weights are displayed in the models depicted.

\subsection{Cybervictimisation, cyberbullying, and QoL}

The standardised, unmediated, direct effect of $\mathrm{T} 1$ cybervictimisation on $\mathrm{T} 4 \mathrm{QoL}$ was significant, with a standardised regression weight of $-0.44(p<0.001), 95 \% \mathrm{Cl}[-0.69,-0.19]$, indicating that as cybervictimisation increased, QoL decreased. The standardised, unmediated direct effect of $\mathrm{T} 1$ cyberbullying on $\mathrm{T} 4 \mathrm{QoL}$ was not significant, with a standardised regression weight of $-0.19(p>05), 95 \% \mathrm{Cl}[-0.56,-0.18]$, indicating that cyberbullying at T1 was not predictive of QoL at T4. Therefore, this was removed from the model and not included in further analyses. This model (Fig. 1) had adequate model fit $[18,20]$ : Bollen-Stine Bootstrap $p=0.33 ; \mathrm{RMSEA}=0.1 ; \mathrm{LO} 90=0.00 ; \mathrm{PCLOSE}=0.25 ; \chi 2 / \mathrm{df}=0.43 ; \mathrm{TLI}=0.96 ; \mathrm{CFI}=0.97$.



Fig. 1 T1 Cybervictimisation predictive of T4 QoL. CV_T1 represents cybervictimisation frequency at time-point 1, QoL represents Quality of Life at time-point 4 


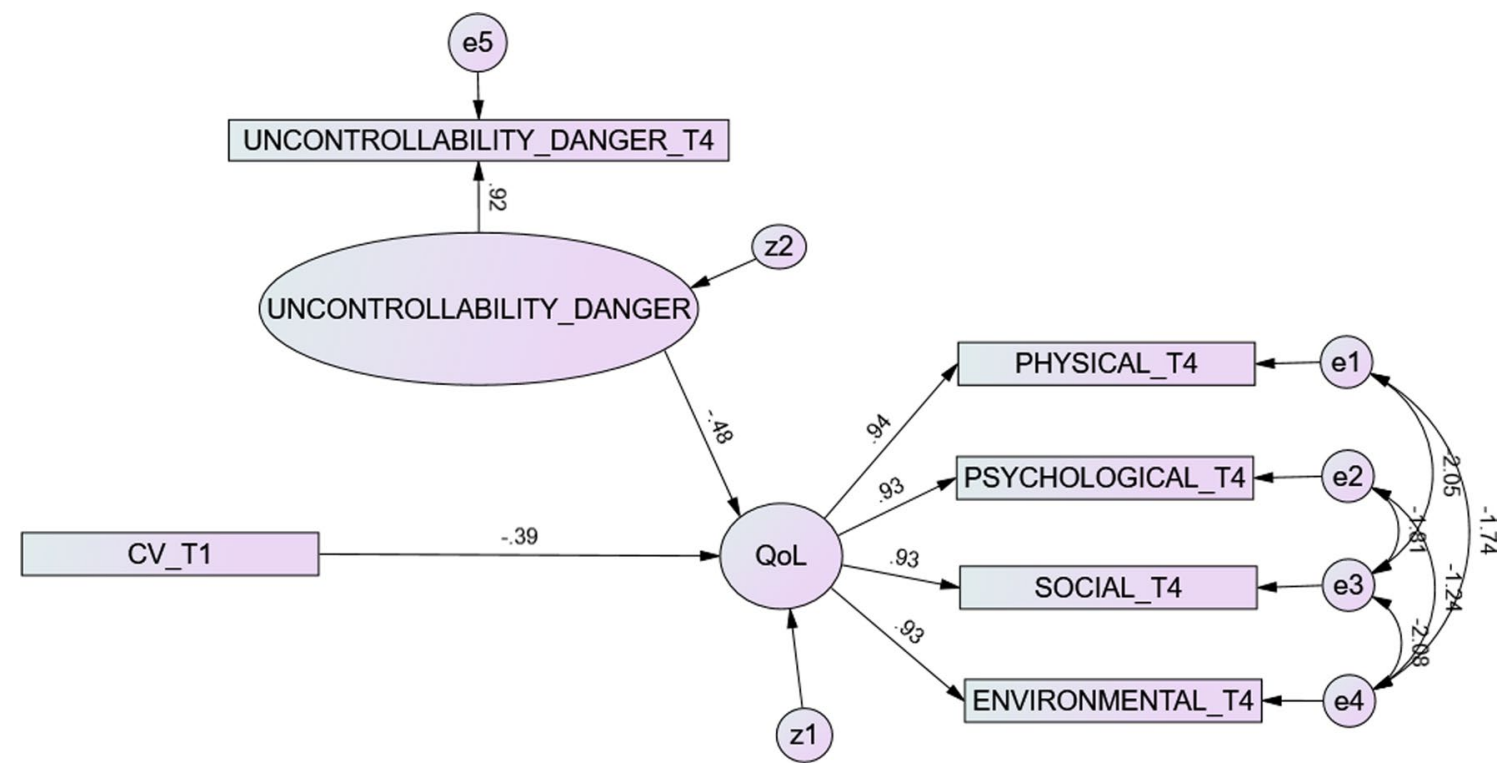

Fig. 2 T1 Cybervictimisation and T4 uncontrollability/danger predictive of T4 QoL. CV_T1 represents cybervictimisation frequency at timepoint 1, QoL represents Quality of Life at time-point 4, UNCONTROLLABILITY_DANGER represents uncontrollability/danger sub-scale of metacognition at time-point 4

\subsection{Positive beliefs mediation}

When 'positive beliefs' was added as a mediator, the standardised effect of T1 cybervictimisation on T4 QoL remained significant, with a standardised regression weight of $-0.43(p<0.001), 95 \% \mathrm{Cl}[-0.68,-0.20]$, indicating that no mediation occurred. The standardised direct effect of T1 cybervictimisation on T4 positive beliefs was not significant, with a standardised regression weight of $0.06(p>05), 95 \% \mathrm{Cl}[-0.19,0.33]$, nor was the standardised direct effect of T4 positive beliefs on T4 QoL, with a standardised regression weight of $-0.13(p>05), 95 \% \mathrm{Cl}[-0.55,0.18]$, confirming no significant associations between these variables.

\subsection{Uncontrollability/danger mediation}

When 'uncontrollability/danger' was added as a mediator, the standardised direct effect of 11 cybervictimisation on T4 QoL remained significant, with a standardised regression weight of $-0.39(p<0.001), 95 \% \mathrm{Cl}[-0.65,-0.15]$, indicating that no mediation occurred. The standardised direct effect of $\mathrm{T} 1$ cybervictimisation on $\mathrm{T} 4$ uncontrollability/danger was not significant, with a standardised regression weight of $0.14(p>05), 95 \% \mathrm{Cl}[-0.10,0.36]$, however the standardised direct effect of T4 uncontrollability/danger on T4 QoL was significant, with a standardised regression weight of -0.48 $(p<0.001), 95 \% \mathrm{Cl}[-0.69,-0.18]$. This indicated that whilst uncontrollability/danger did not mediate the association between cybervictimisation and QoL, and cybervictimisation was not predictive of uncontrollability/danger, both uncontrollability/danger and cybervictimisation were predictive of QoL. This model (Fig. 2) had adequate model fit [18, 20]: Bollen-Stine Bootstrap $p=0.399 ; \mathrm{RMSEA}=0.073 ; \mathrm{LO} 90=0.000 ; \mathrm{PCLOSE}=0.346 ; \chi 2 / \mathrm{df}=1.232 ; \mathrm{TLI}=0.973 ; \mathrm{CFI}=0.980$.

\subsection{Cognitive confidence mediation}

When cognitive confidence was added as a mediator, the standardised direct effect of T1 cybervictimisation on T4 QoL was no longer significant, with a standardised regression weight of $-0.22(p>0.05), 95 \% \mathrm{Cl}[-0.53,-0.14]$. The standardised direct effect of 11 cybervictimisation on T4 cognitive confidence was significant, with a standardised regression weight of $0.46(p<0.001), 95 \% \mathrm{Cl}[0.13,0.69]$, and the standardised direct effect of T4 cognitive confidence on T4 QoL was significant, with a standardised regression weight of $-0.63(p<0.001), 95 \% \mathrm{Cl}[-0.93,-0.44]$. In addition, T1 cybervictimisation had a significant indirect effect on T4 QoL, with a standardised regression weight of $-0.29(p<0.001), 95 \%$ 


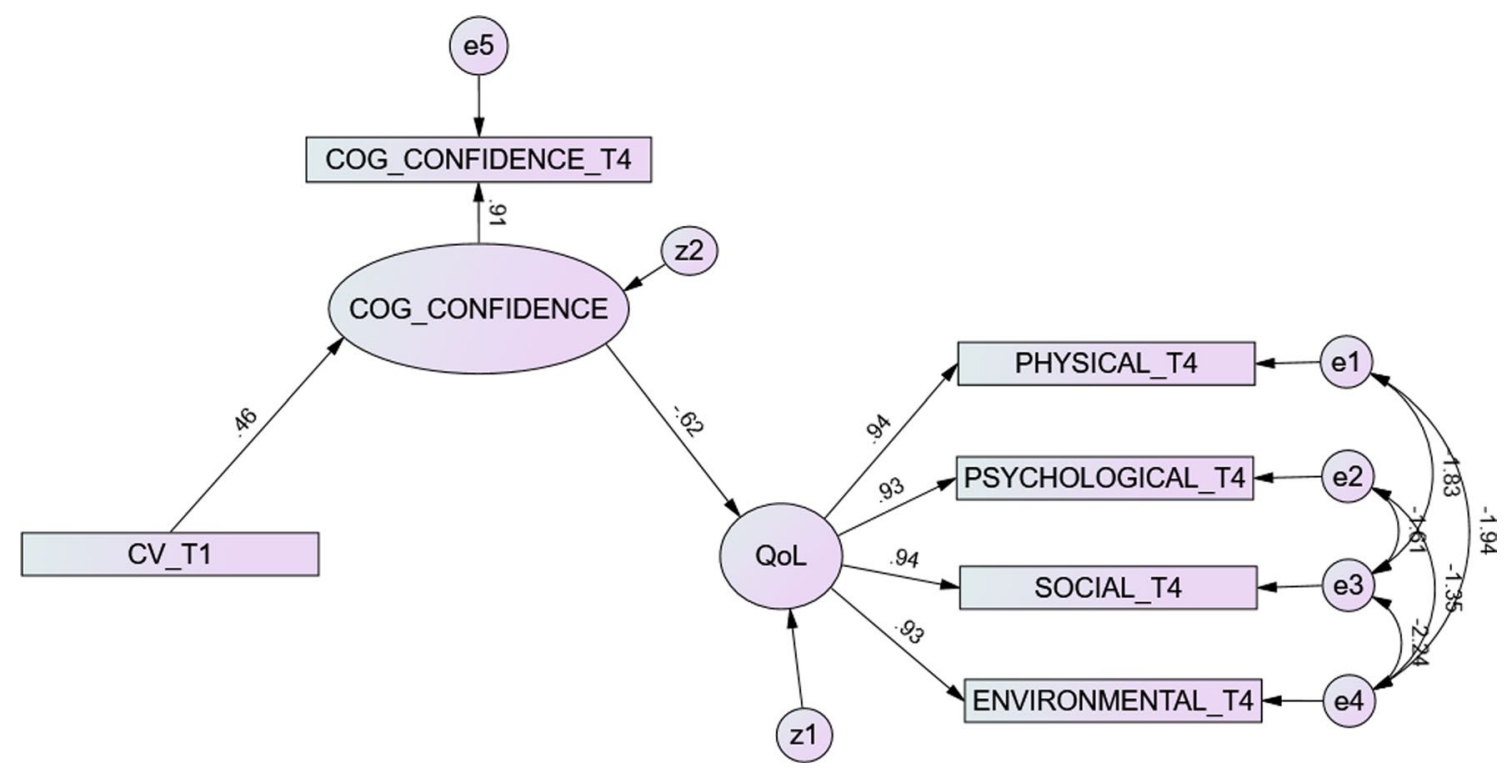

Fig. 3 Cognitive confidence mediation between cybervictimisation and QoL. CV_T1 represents cybervictimisation frequency at time-point 1, QoL represents Quality of Life at time-point 4, COG_CONFIDENCE represents cognitive confidence sub-scale of metacognition at timepoint 4

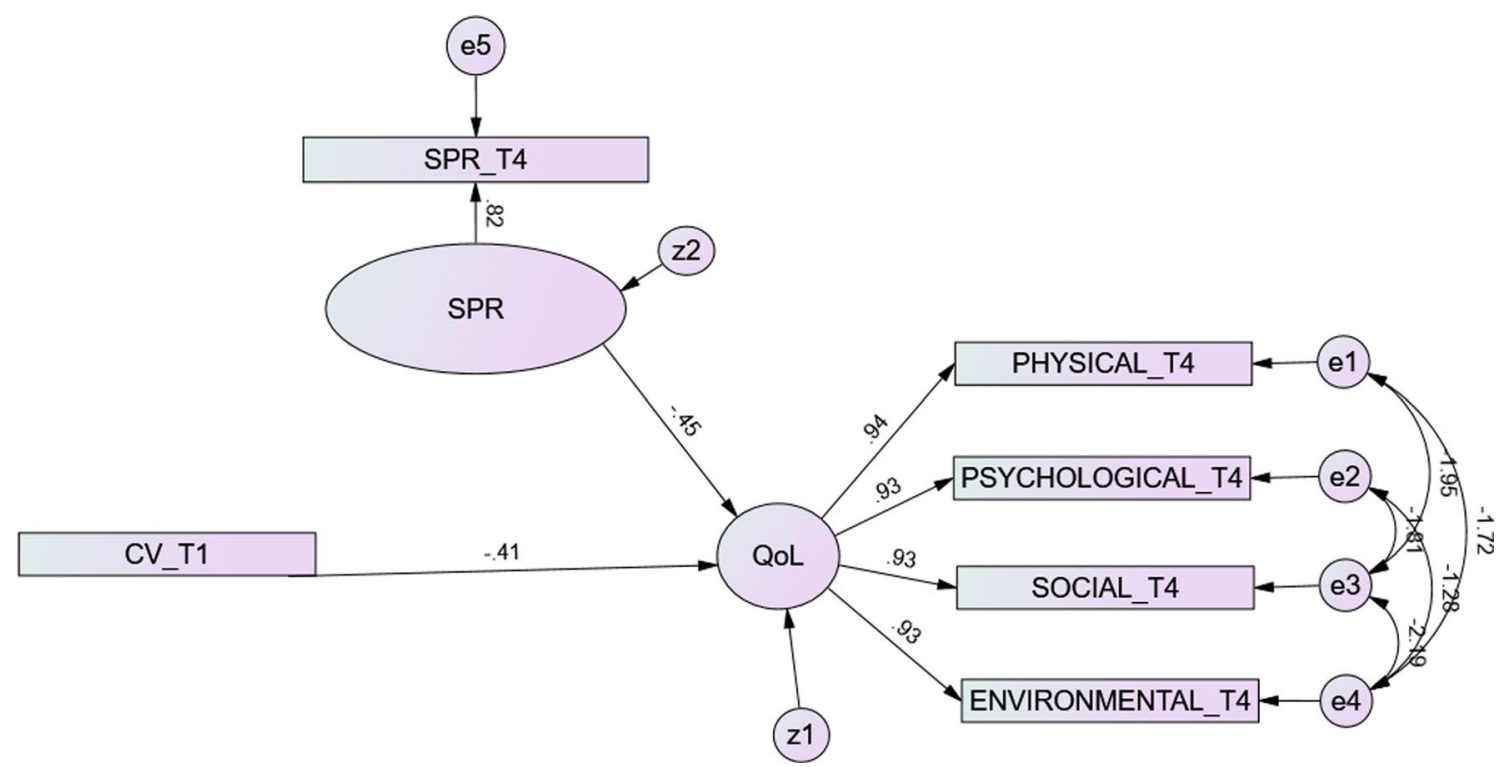

Fig. 4 T1 Cybervictimisation and T4 SPR predictive of T4 QoL. CV_T1 represents cybervictimisation frequency at time-point 1, QoL represents Quality of Life at time-point 4, SPR represents superstition, punishment, and responsibility sub-scale of metacognition at time-point 4

$\mathrm{Cl}[-0.55,-0.08]$, indicating that partial mediation may have occurred. This model (Fig. 3) had adequate model fit [18, 20]: Bollen-Stine Bootstrap $p=0.354 ; \mathrm{RMSEA}=0.117 ; \mathrm{LO} 90=0.000 ; \mathrm{PCLOSE}=0.150 ; \chi 2 / \mathrm{df}=1.585 ; \mathrm{TLI}=0.936 ; \mathrm{CFI}=0.953$.

\subsection{Superstition, punishment, and responsibility mediation}

When SPR was added as a mediator, the standardised direct effect of T1 cybervictimisation on T4 QoL remained significant, with a standardised regression weight of $-0.41(p<0.001), 95 \% \mathrm{Cl}[-0.64,-0.20]$, indicating that no mediation occurred. The standardised direct effect of T1 cybervictimisation on T4 SPR was not significant, with a standardised regression weight of $0.09(p>05), 95 \% \mathrm{Cl}[-0.23,0.48]$, however the standardised direct effect of T4 SPR on T4 QoL was 
significant, with a standardised regression weight of $-0.45(p<0.001), 95 \% \mathrm{Cl}[-0.77,-0.18]$. This indicates that whilst SPR did not mediate the association between cybervictimisation and QoL, and cybervictimisation was not predictive of SPR, both SPR and cybervictimisation were predictive of QoL. This model (Fig. 4) had adequate model fit [18, 20]: BollenStine Bootstrap $p=0.249 ; \mathrm{RMSEA}=0.114 ; \mathrm{LO} 90=0.000 ; \mathrm{PCLOSE}=0.161 ; \chi 2 / \mathrm{df}=1.558 ; \mathrm{TLI}=0.935 ; \mathrm{CFI}=0.952$.

\subsection{Cognitive self-consciousness mediation}

When cognitive self-consciousness was added as a mediator, the standardised effect of T1 cybervictimisation on T4 QoL remained significant, with a standardised regression weight of $-0.44(p<0.001), 95 \% \mathrm{Cl}[-0.70,-0.18]$, indicating that no mediation occurred. The standardised direct effect of T1 cybervictimisation on T4 cognitive self-consciousness was not significant, with a standardised regression weight of $-0.12(p>05), 95 \% \mathrm{Cl}[-.39,0.15]$, nor was the standardised direct effect of T4 cognitive self-consciousness on T4 QoL, with a standardised regression weight of $-0.018(p>05), 95 \%$ $\mathrm{Cl}[-0.24,0.23]$, confirming no significant associations between these variables.

\section{Discussion}

To our knowledge, this paper is the first to longitudinally explored associations between cyberbullying, cybervictimisation, metacognitive beliefs and QoL. This paper investigated whether different forms of metacognition mediate the association between cyberbullying and cybervictimisation on QoL, over time. SEM showed that whilst cybervictimisation negatively predicted QoL over time, cyberbullying did not. These results suggest that as cybervictimisation increases, QoL decreases, supporting the first of our hypotheses. In other words, the more frequently young people were cybervictimised, the lower their QoL scores were one year later. These findings are consistent with previous research, which suggests that peer victimisation is associated with decreased QoL $[13,14]$. However, our findings are unique in that they indicate that cybervictimisation can impact QoL one year later in young people as young as 12 years of age.

Mediation analysis through SEM showed that not all subscales of metacognition were predictive of QoL (only cognitive confidence, uncontrollability/danger and superstition, punishment, and responsibility were), nor were they associated with cybervictimisation or cyberbullying. Only one subscale of metacognition (cognitive confidence) acted as a mediator between cybervictimisation and QoL and, thus, supporting our second and third hypotheses. More specifically, higher scores of cognitive confidence indicates reduced confidence in memory; therefore, those who were more frequently cybervictimised had reduced confidence in their memory, which resulted in lower QoL. Whilst limited research has examined the links between metacognition and cyberbullying, studies have found that low cognitive confidence in memory was associated with obsessive-compulsive checking, trait anxiety, and worry [39]. Research has demonstrated that low cognitive confidence and distrust in one's memory and attention is a factor in OCD samples [27]. Further, low confidence has been found to interact with experiencing daily hassles, including social rejection and interpersonal conflicts, to predict anxiety [46]. Potentially for cyberbullying victims, distrust in their memory and obsessive-checking (e.g., checking and re-reading bullying messages), as well as, daily hassles that may encompass social rejection and conflicts, are relevant factors that require further research. A recent study reported that cybervictims repeatedly checked, and engaged in over thinking about perpetrators bullying posts, and that each encounter with the content triggered victims to re-experience the initial distress and maintain feelings of anguish about the event [9]. However, our results are the first to provide longitudinal evidence of a relationship between metacognitive beliefs and experiences of cybervictimisation, and that cognitive confidence could influence QoL as a result.

Whilst both uncontrollability/danger and SPR did not mediate the association between cybervictimisation and QoL, and were not significantly associated with cybervictimisation, they were negatively associated with QoL, indicating that as these metacognitive beliefs increased, QoL decreased. In other words, young people who felt a higher need for control over their thoughts and being responsible for the negative consequences of not doing so (SPR), and had beliefs that worry is harmful, dangerous, and uncontrollable (uncontrollability/danger) had lower QoL, and experiences of cybervictimisation did not predict these beliefs (but independently predicted QoL). Finally, positive beliefs and cognitive self-consciousness showed no associations with cybervictimisation and QoL through SEM and had no effect on the negative association between cybervictimisation and QoL. This indicates that the belief that worrying may be helpful in preventing or avoiding problems in the future, and the extent to which a person is aware of, and focuses on, their thinking, had no significant association with cybervictimisation or QoL in the current sample. These results support previous research which suggest that metacognitive beliefs are associated with pathological worry, obsessive-compulsive 
symptoms, and rumination in depression $[5,28,35,46]$. These associations are important as research shows that anxiety and depression may result from difficulty controlling repetitive negative thinking processes [40].

\section{Limitations and future directions}

There are several limitations associated with this study that require clarification. Firstly, the sample size when considering each time-point individually is small, and so findings should be interpreted taking this into consideration. Future research should aim to replicate these models with larger sample sizes, also taking into consideration age and gender differences. Our paper adds value to the current literature as it is the first study to have a sample made entirely of young people aged 12 years. As the study continues, we will be in a unique position to track how these same individuals have changed over time.

Secondly, findings regarding cyberbullying (in contrast to cybervictimisation) were not significant. This could be for any number of reasons, such as social desirability bias. Participants may have been concerned about disclosing in the self-report having cyberbullied other people. This is commonly encountered in cyberbullying research and is difficult to address. It is hoped that replication of these measures over time from LABS will account for this bias.

This research is significant as our findings highlight the detrimental impact that cybervictimisation experiences can have on QoL in young people over time, and that some forms of metacognitive beliefs can also impact QoL. Specifically, cognitive confidence may be significantly reduced by experiences of cyberbullying. These findings can inform educators and health professionals on the importance of metacognition in regard to QoL over time, particularly in those who experience cybervictimisation.

\section{Conclusion}

This paper examined the associations between cyberbullying and cybervictimisation, metacognition, and quality of life, and is one of the first to examine these associations over time. Whilst previous research has examined links between metacognition and victimisation, and metacognition and QoL, little research has examined how the three variables may relate to each other, especially longitudinally. The current findings have indicated that some dimensions of metacognition can predict QoL. In particular, uncontrollability/danger and SPR both influenced QoL as an outcome, though were not linked to cybervictimisation. Cognitive confidence, however, partially mediated the association between cybervictimisation and QoL, indicating that whilst cybervictimisation experiences are associated with higher cognitive confidence scores, low confidence scores can in turn impact QoL. These findings can inform future research, as well as educators and health professionals around the impact metacognitive beliefs can have on QoL, particularly in light of cybervictimisation experiences over time.

Acknowledgements Many thanks to the young people who gave their time to participate in this research. Thank you also to the LABS research assistants and students.

Author contributions LM wrote the main manuscript text. LM completed the data analyses. All authors contributed to drafts of the manuscript. All authors reviewed the manuscript.

Funding This research is supported by a grant from the Australian Commonwealth Government's 'Prioritizing Mental Health Initiative' (201819), 4-7R154BP.

Data availability The data that support the findings of this study are available on request from the corresponding author. The data are not publicly available due to privacy or ethical restrictions.

Declarations

Ethics approval and consent for publication The University of the Sunshine Coast (USC) Human Research Ethics Committee approved the study (\#A181064) and informed consent was obtained from all participants and caregivers prior to participating.

Competing interests The authors have no conflicts of interest to declare.

Open Access This article is licensed under a Creative Commons Attribution 4.0 International License, which permits use, sharing, adaptation, distribution and reproduction in any medium or format, as long as you give appropriate credit to the original author(s) and the source, 
provide a link to the Creative Commons licence, and indicate if changes were made. The images or other third party material in this article are included in the article's Creative Commons licence, unless indicated otherwise in a credit line to the material. If material is not included in the article's Creative Commons licence and your intended use is not permitted by statutory regulation or exceeds the permitted use, you will need to obtain permission directly from the copyright holder. To view a copy of this licence, visit http://creativecommons.org/licenses/by/4.0/.

\section{References}

1. Arbuckle JL. Amos for Windows, Version 26.0. New York: IBM Corp; 2019.

2. Blanc J, Boyer L, Le Coz P, Auquier P. Metacognition: towards a new approach to quality of life. Qual Life Res. 2014;23(2):467-75. https:// doi.org/10.1007/s11136-013-0485-7.

3. Campbell M, Spears B, Slee P, Butler D, Kift S. Victims' perceptions of traditional and cyberbullying, and the psychosocial correlates of their victimisation. Emot Behav Diffic. 2012;17(3-4):389-401.

4. Cartwright-Hatton S, Mather A, Illingworth V, Brocki J, Harrington R, Wells AJJ. Development and preliminary validation of the Metacognitions Questionnaire-Adolescent Version. J Anxiety Disorders. 2004;18(3):411-22.

5. Cartwright-Hatton S, Wells A. Beliefs about worry and intrusions: the meta-cognitions questionnaire and its correlates. J Anxiety Disord. 1997;11(3):279-96.

6. Chen K-H, Wu C-H, Yao G. Applicability of the WHOQOL-BREF on early adolescence. Soc Indic Res. 2006;79(2):215-34.

7. Chin WW. Commentary: issues and opinion on structural equation modeling. MIS Quarterly. 1998;22:1.

8. Corportation I. IBM SPSS Statistics for Windows, Version 26.0. New York: IBM Corp; 2019.

9. Dennehy R, Meaney S, Cronin M, Arensman E. The psychosocial impacts of cybervictimisation and barriers to seeking social support: Young people's perspectives. Children Youth Serv Rev. 2020;111:104872. https://doi.org/10.1016/j.childyouth.2020.104872.

10. Ellis DM, Hudson J, et al. Test of the metacognitive model of generalized anxiety disorder in anxiety-disordered adolescents. J Exp Psychopathol. 2011;2(1):28-43.

11. Fahy AE, Stansfeld SA, Smuk M, Smith NR, Cummins S, Clark C. Longitudinal associations between cyberbullying involvement and adolescent mental health. J Adolesc Health. 2016;59(5):502-9. https://doi.org/10.1016/j.jadohealth.2016.06.006.

12. Gini G, Marino C, Spada MM. The role of metacognitions and thinking styles in the negative outcomes of adolescents' peer victimization. Violence Vict. 2019;34(5):752-69.

13. González-Cabrera J, León-Mejía A, Beranuy M, Gutiérrez-Ortega M, Álvarez-Bardón A, Machimbarrena JM. Relationship between cyberbullying and health-related quality of life in a sample of children and adolescents. Qual Life Res. 2018;27(10):2609-18.

14. González-Cabrera J, Machimbarrena JM, Ortega-Barón J, Álvarez-Bardón A. Joint association of bullying and cyberbullying in healthrelated quality of life in a sample of adolescents. Qual Life Res. 2019;1:1-12.

15. Group, W. The World Health Organization quality of life assessment (WHOQOL): position paper from the World Health Organization. Soc Sci Med. 1995;41(10):1403-9.

16. Haynie D, Nansel T, Eitel P, Crump A, Saylor K, Yu K, Simons-Morton B. Bullies, victims, and bully/victims: distinct groups of at-risk youth JEarly Asolesc. 2001;21(1):29-49. https://doi.org/10.1177/0272431601021001002.

17. Holmes-Smith P. Structural Equation Modelling (Using AMOS): From the Fundamentals to Advanced Topics. School Research Evaluation and Measurement Services; 2015.

18. Hu L, Bentler PM. Cutoff criteria for fit indexes in covariance structure analysis: conventional criteria versus new alternatives. Struct Equ Modeling. 1999;6(1):1-55.

19. Kim S, Georgiades K, Comeau J, Vitoroulis I, Boyle MH. The association between cyberbullying victimization and adolescent mental health: a comparative study between traditional types of bullying versus cyberbullying. J Am Acad Child Adolesc Psychiatry. 2016;55(10):S160.

20. Kline RB. Principles and practice of structural equation modeling. New York: Guilford publications; 2015.

21. Kowalski RM, Limber SP. Psychological, physical, and academic correlates of cyberbullying and traditional bullying. J Adolesc Health. 2013;53(1):S13-20. https://doi.org/10.1016/j.jadohealth.2012.09.018.

22. Kranzler A, Young JF, Hankin BL, Abela JR, Elias MJ, Selby EAJJ, o. C. C., \& Psychology, A. Emotional awareness: a transdiagnostic predictor of depression and anxiety for children and adolescents. J Clin Child Adolesc Psychol. 2016;45(3):262-9.

23. Le HT, Nguyen HT, Campbell MA, Gatton ML, Tran NT, Dunne MP. Longitudinal associations between bullying and mental health among adolescents in Vietnam. Int J Public Health. 2017;62(Suppl 1):51-61. https://doi.org/10.1007/s00038-016-0915-8.

24. McEvoy P. Metacognitive therapy for anxiety disorders: a review of recent advances and future research directions. Curr Psychiatry Rep. 2019:21:29.

25. McLoughlin L, Spears B, Taddeo C. The importance of social connection for cybervictims: how connectedness and technology could promote mental health and wellbeing in young people. Int J Emot Educ. 2018;10(1):5-24.

26. McLoughlin LT, Spears BA, Taddeo CM, Hermens DF. Remaining connected in the face of cyberbullying: why social connectedness is important for mental health. Psychol Schools. 2019;56(6):945-58. https://doi.org/10.1002/pits.22232.

27. Ouellet-Courtois C, Wilson S, O'Connor K. Cognitive confidence in obsessive-compulsive disorder: a systematic review and meta-analysis. J Obsess Compuls Related Disorders. 2018;19:77-86. https://doi.org/10.1016/j.jocrd.2018.08.003.

28. Papageorgiou C, Wells A. Metacognitive beliefs about rumination in recurrent major depression. Cogn Behav Pract. 2001;8(2):160-4. https://doi.org/10.1016/S1077-7229(01)80021-3

29. Patchin JW, Hinduja S. Bullies move beyond the schoolyard: a preliminary look at cyberbullying. Youth Violence Juvenile Justice. 2006;4(2):148-69. https://doi.org/10.1177/1541204006286288.

30. Schultze-Krumbholz A, Scheithauer H. Measuring Cyberbullying and Cybervictimisation by Using Behavioral Categories - The Berlin Cyberbullying Cybervictimisation Questionnaire (BCyQ); 2009.

31. Schultze-Krumbholz A, Scheithauer H. The Berlin Cyberbullying-Cybervictimization Questionnaire (BCyQ). Freie Universität Berlin: Unpublished Questionnaire; 2011. 
32. Smith PK, Mahdavi J, Carvalho M, Fisher S, Russell S, Tippett N. Cyberbullying: its nature and impact in secondary school pupils. J Child Psychol Psychiatry. 2008;49(4):376-85. https://doi.org/10.1111/j.1469-7610.2007.01846.x.

33. Spears BA, Taddeo CM, Daly AL, Stretton A, Karklins LT. Cyberbullying, help-seeking and mental health in young Australians: implications for public health. Int J Public Health. 2015;60(2):219-26. https://doi.org/10.1007/s00038-014-0642-y.

34. Stevens J. Applied multivariate statistics for the social sciences. New Jersey: L. Erlbaum Associates Inc.; 1986.

35. Tajrishi KZ, Mohammadkhani S, Jadidi F. Metacognitive beliefs and negative emotions. Procedia Soc Behav Sci. 2011;30:530-3. https:// doi.org/10.1016/j.sbspro.2011.10.103.

36. Thomas HJ, Connor JP, Lawrence DM, Hafekost JM, Zubrick SR, Scott JG. Prevalence and correlates of bullying victimisation and perpetration in a nationally representative sample of Australian youth. Aust N Z J Psychiatry. 2017;51(9):909-20. https://doi.org/10.1177/00048 67417707819.

37. van Geel M, Vedder P, Tanilon J. Relationship between peer victimization, cyberbullying, and suicide in children and adolescents: a metaanalysis. JAMA Pediatr. 2014;168(5):435-42. https://doi.org/10.1001/jamapediatrics.2013.4143.

38. Völlink T, Bolman CAW, Eppingbroek A, Dehue F. Emotion-focused coping worsens depressive feelings and health complaints in cyberbullied children. J Criminol. 2013;2013:1-10. https://doi.org/10.1155/2013/416976.

39. Wells A. Emotional disorders and metacognition: innovative cognitive therapy. New York: Wiley; 2002.

40. Wells A. The metacognitive model of worry and generalised anxiety disorder. Worry and its psychological disorders: Theory Assessment and Treatment. 2006;1:179-99.

41. Wells A. Metacognitive therapy for anxiety and depression. New York: Guilford press; 2011.

42. Wells A. Advances in metacognitive therapy. J Int J Cogn Ther. 2013;6(2):186-201.

43. Wells A, Capobianco L. Metacognition. In J. S. Abramowitz \& S. M. Blakey (Eds.), Clinical handbook of fear and anxiety: Maintenance processes and treatment mechanisms. (pp. 171-182). American Psychological Association; 2020. https://doi.org/10.1037/0000150-010

44. Wells A, Cartwright-Hatton SJB, r., \& therapy. A short form of the metacognitions questionnaire: properties of the MCQ-30. J Behav Res. 2004;42(4):385-96.

45. Wells A, Matthews G. Modelling cognition in emotional disorder: the S-REF model. Behav Res Ther. 1996;34(11):881-8. https://doi.org/ 10.1016/S0005-7967(96)00050-2.

46. Yılmaz AE, Gençöz T, Wells A. The temporal precedence of metacognition in the development of anxiety and depression symptoms in the context of life-stress: a prospective study. J Anxiety Disord. 2011;25(3):389-96.

Publisher's Note Springer Nature remains neutral with regard to jurisdictional claims in published maps and institutional affiliations. 